\title{
Editorial
}

\section{Laser and Ion Beams Interactions with Materials}

\author{
Xiaotao Zu, ${ }^{1,2}$ Kai Sun, ${ }^{3}$ Wanguo Zheng, ${ }^{4}$ and Haiyan Xiao ${ }^{2}$ \\ ${ }^{1}$ Institute of Fundamental and Frontier Sciences, University of Electronic Science and Technology of China, Chengdu 610054, China \\ ${ }^{2}$ School of Physical Electronics, University of Electronic Science and Technology of China, Chengdu 610054, China \\ ${ }^{3}$ Department of Materials Science and Engineering, University of Michigan, Ann Arbor, MI 48109, USA \\ ${ }^{4}$ Research Center of Laser Fusion, China Academy of Engineering Physics, Mianyang 621900, China
}

Correspondence should be addressed to Xiaotao Zu; xtzu@uestc.edu.cn

Received 13 October 2014; Accepted 13 October 2014; Published 22 December 2014

Copyright (C) 2014 Xiaotao Zu et al. This is an open access article distributed under the Creative Commons Attribution License, which permits unrestricted use, distribution, and reproduction in any medium, provided the original work is properly cited.

Laser and ion beams, as important energetic sources, provide the ability to accurately deliver large amounts of energy into confined regions of a material and induce changes to the local geometrical and electronic structure, consequently affecting materials properties and behaviors in a given application. They have become indispensible and powerful tools in the fields ranging from materials science to biomedicine and can be used for various applications, including surface modification, thin-film deposition, materials processing, and nanostructure formation. Understanding of laser and ion beams interactions with materials will enable scientists to explore materials structures and properties under extreme conditions like intense laser and irradiation, to manipulate and engineer materials properties, as well as to design novel and innovative materials and devices with enhanced and improved performance or desirable properties.

This special issue encompasses the fundamentals of lasermaterial and ion beam-material interactions and the application of laser and ion beams for modifying, processing, and manufacturing of electronic, photonic, optical, mechanical, and energy materials. It covers the intense laser beam and operation environment induced damage and damage growth behaviors of optical materials and components such as fused silica glass and KDP crystals in the large high power laser facilities, the damage mitigation methods and technologies by using ultraviolet or infrared laser conditioning, laser cladding and laser cleaning, and the on-line detection and control of contaminations in the laser facilities. Ion beam interaction with materials mainly focuses on the neutron and helium ion irradiation of metals such as aluminum and zirconium. Both experimental and theoretical investigations are included.
Fused silica glass and KDP crystals have excellent ultraviolet transparency, optical homogeneity, and good damage resistance, so they are usually used as optical window materials in the large high power laser systems such as National Ignition Facility (NIF) in USA, Laser Megajoule (LMJ) in France, and Shenguang-III (SG-III) facility in China. However, the effects of laser interaction with materials may become obvious with the increasing laser fluency, including temperature rise, expansion, strain, distortion, nonlinear absorption, and transmittance change. As the laser intensity increases further, some nonreversible changes may occur in the optical materials or components, such as melting and evaporation, material softening and bending, pitting, cracking, and shattering. Interaction between laser and optical components can cause permanent changes which are called laser-induced damage (LID) in the material. The laser-induced damage threshold (LIDT) is the level of laser radiation which initiates some alteration of the optics under examination. At present, how to improve the LIDT becomes a very important issue in the fields of laser design, optical engineering, optical materials, contamination control, and so forth. In this special issue, the investigations on laser induced point defects, damage initiation, and damage growth behaviors of fused silica. The most important several methods to improve the LIDT of fused silica and KDP materials are also reported in this special issue, including ultraviolet laser conditioning, infrared $\mathrm{CO}_{2}$ laser conditioning and damage mitigation, and surface contaminant detection based on nanostructured sensors and contaminant control. Three papers about laser application are the laser ignition of triaminotrinitrobenzene (TATB) explosives, laser cladding of blades materials to improve its 
water erosion resistance, and laser cleaning of low carbon steel alloys, respectively.

As a conventional and important topic, the radiation effects of ion beams and neutrons on the nuclear materials always attract many interests. In this special issue, the structure and properties of helium in $\alpha$-zirconium are studied by the first principles and the evolution of helium with temperature in the neutron-irradiated ${ }^{10} \mathrm{~B}$-doped aluminum is studied by small-angle $\mathrm{X}$-ray scattering.

\section{Acknowledgment}

We would like to thank all contributors for their hard work and patience in bringing out this special issue. We also expect that the special issue will stimulate the continuing efforts to deepen the investigation on the laser and ion beams interactions with materials.

Xiaotao Zu

Kai Sun

Wanguo Zheng

Haiyan Xiao 

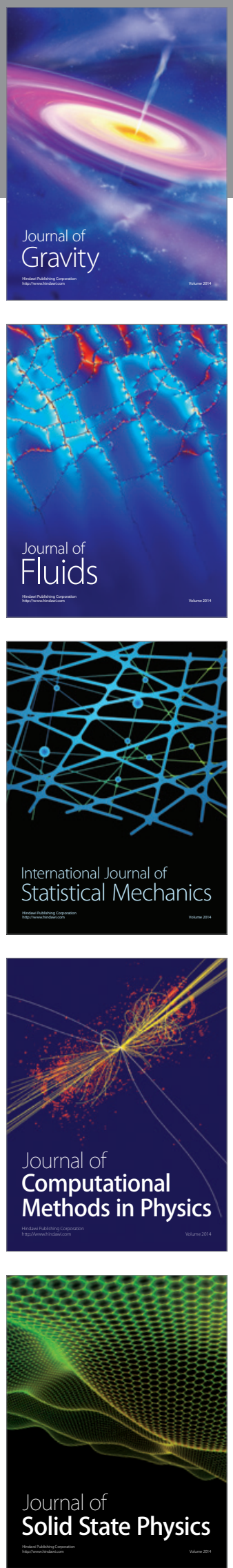

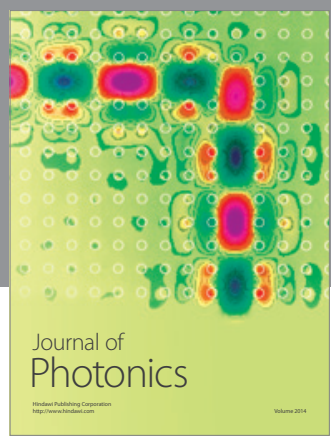

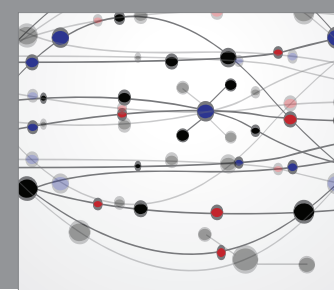

The Scientific World Journal

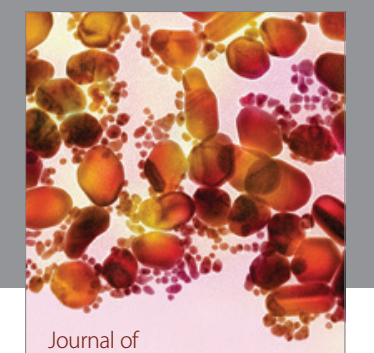

Soft Matter
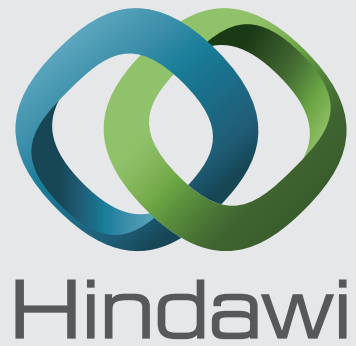

Submit your manuscripts at

http://www.hindawi.com
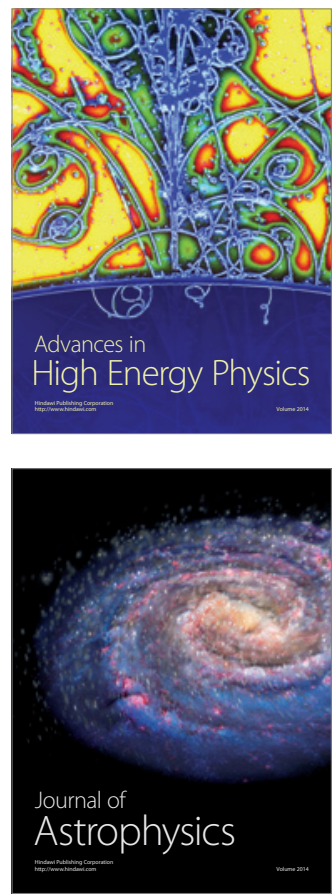
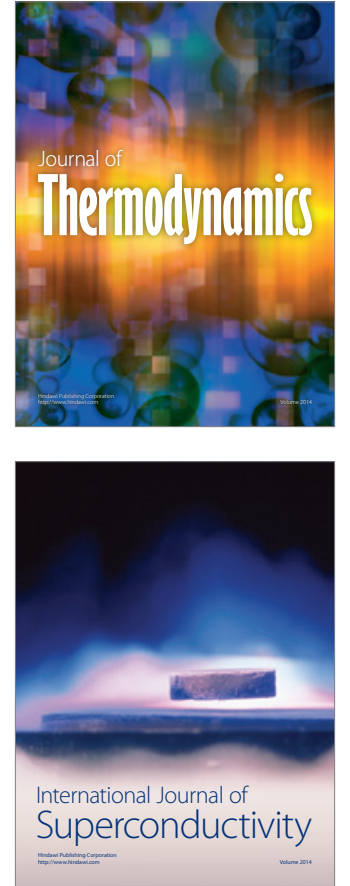
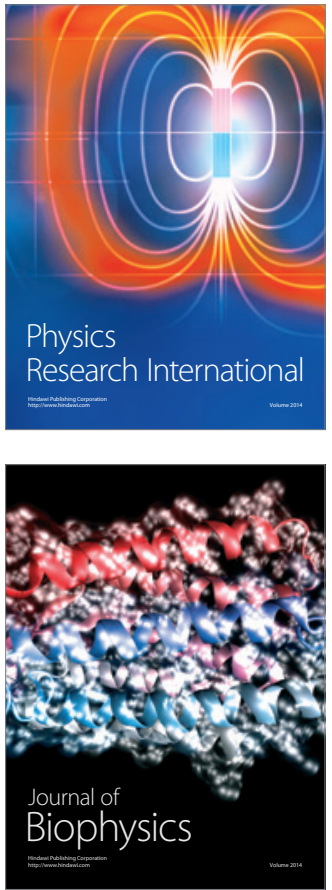
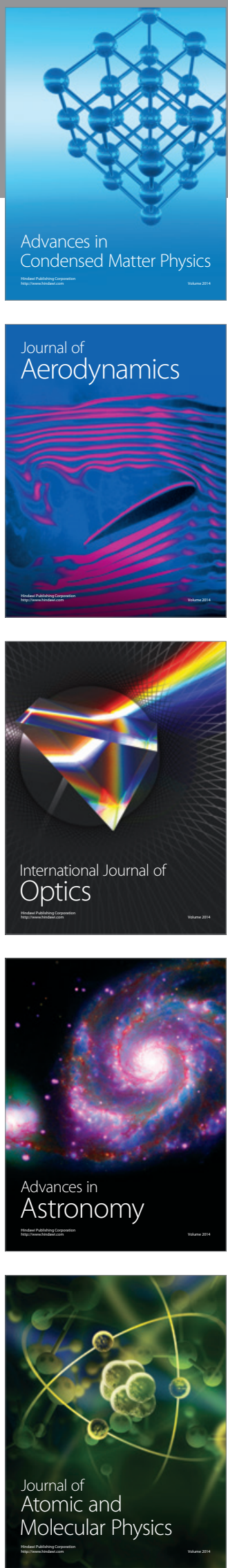